\title{
RESEARCH BRIEF \\ Prevalence of Self-Reported Intake of Sugar- Sweetened Beverages Among US Adults in 50 States and the District of Columbia, 2010 and 2015
}

\author{
Jennifer R. Chevinsky, MD, MPH ${ }^{1,2}$; Seung Hee Lee, $\mathrm{PhD}^{1}$; Heidi M. Blanck, $\mathrm{PhD}^{1}$; Sohyun Park, $\mathrm{PhD}^{1}$
}

\begin{abstract}
Accessible Version: www.cdc.gov/pcd/issues/2021/20_0434.htm
Suggested citation for this article: Chevinsky JR, Lee SH, Blanck HM, Park S. Prevalence of Self-Reported Intake of SugarSweetened Beverages Among US Adults in 50 States and the District of Columbia, 2010 and 2015. Prev Chronic Dis 2021; 18:200434. DOI: https://doi.org/10.5888/pcd18.200434.
\end{abstract}

\section{PEER REVIEWED}

\section{Summary}

What is already known about this topic?

Frequent intake of sugar-sweetened beverages (SSBs) is associated with adverse health consequences. SSB intake differs by geographical region and sociodemographic characteristics.

\section{What is added by this report?}

We report SSB intake by state for all 50 states and the District of Columbia along with notable geographic and sociodemographic differences.

What are the implications for public health practice?

Efforts to decrease SSB intake could consider sociodemographic and geographic differences in SSB intake to inform design of interventions.

\section{Abstract}

Frequent intake of sugar-sweetened beverages (SSBs) is associated with adverse health outcomes, including obesity, type 2 diabetes, and cardiovascular disease. We used combined data from the 2010 and 2015 National Health Interview Survey to examine the prevalence of SSB intake among US adults in all 50 states and the District of Columbia. Approximately two-thirds of adults reported consuming SSBs at least daily, including more than 7 in 10 adults in Hawaii, Arkansas, Wyoming, South Dakota, Connecticut, and South Carolina, with significant differences in sociodemographic characteristics. Efforts to decrease SSB consumption could consider the sociodemographic and geographic differences in SSB intake when designing equitable interventions.

\section{Objective}

Sugar-sweetened beverages (SSBs) are a leading source of added sugars in the US diet and are associated with obesity, type 2 diabetes, heart disease, kidney disease, nonalcoholic fatty liver disease, and tooth decay (1-4). SSBs, which are sweetened with various forms of added sugars, include regular soda, sweetened fruit drinks, sports/energy drinks, and sweetened coffee/tea drinks (5). Previous studies reported geographic differences in SSB intake (6-8). However, no study has reported SSB intake for every state. We assessed the prevalence of SSB intake among US adults by sociodemographic characteristics for all 50 states and the District of Columbia by using National Health Interview Survey (NHIS) data.

\section{Methods}

NHIS is a nationally representative, cross-sectional household survey conducted by the National Center for Health Statistics (NCHS) that uses in-person interviews. The Cancer Control Supplement (CCS), which contains dietary intake information, was administered both in 2010 and in 2015 and was approved by the NCHS Research Ethics Review Board. We used nationally weighted data from combined 2010 and 2015 NHIS CCS to examine the prevalence of consuming SSBs 1 or more times daily among 56,260 US adults aged 18 or older. Data were combined to increase the sample size and reduce the variability associated with state estimates. This study required the use of restricted NHIS files for state estimates and categorizing metropolitan status available through the NCHS Research Data Center. SSB intake was based on survey respondents' answers to 4 questions asking about intake frequency over the past month of regular soda, sweetened fruit drinks, sports/energy drinks, and sweetened coffee/tea drinks $(9,10)$. Sweetened fruit drinks and sweetened coffee/tea drinks included drinks that were presweetened in addition to drinks that were sweetened at home by adding sugar. Adults responded with intake frequency per day, week, or month for each beverage type. Weekly and monthly intake frequency for each type of beverage was converted to daily intake frequency by dividing by 7 or 30 , re- 
spectively. To calculate frequency of total daily SSB intake, we summed responses from intake of regular soda, sweetened fruit drinks, sports/energy drinks, and sweetened coffee/tea drinks. SSB categories and frequency cutoff of once per day were used, consistent with previous studies $(6,7)$. Differences in respondent characteristics were assessed by $\chi^{2}$ tests $(P<.05)$. Prevalence estimates were calculated for SSB categories and by state for all 50 states and the District of Columbia. Analyses were conducted with SAS-callable SUDAAN, version 9.0 (RTI) to account for a complex survey design and sampling weights.

\section{Results}

Overall, $63.0 \%$ of US adults reported consuming SSBs 1 or more times daily in combined 2010 and 2015 NHIS CCS data (Table 1). US adults reported consuming the following 1 or more times daily, by beverage type: sweetened coffee/tea drinks, 39.5\%; regular soda, $19.5 \%$; fruit drinks, 5.7\%; and sports/energy drinks, 5.5\%. Among sociodemographic categories with significant differences overall, the prevalence of SSB intake was highest among adults aged 18 to $24(65.0 \%)$ and 25 to $39(65.4 \%)$, men $(66.1 \%)$, Hispanic respondents $(70.1 \%)$, people with less than a high school education (69.8\%), people with an annual household income less than $\$ 35,000(66.0 \%)$, people residing in nonmetropolitan areas $(65.0 \%)$, and people residing in the Northeast census region $(67.0 \%)$. The prevalence of SSB intake did not significantly differ by marital status.

By state, SSB intake of 1 or more times daily ranged from $44.5 \%$ in Alaska to $76.4 \%$ in Hawaii. These 6 states had a prevalence of daily SSB intake of $70.0 \%$ or more: Hawaii (76.4\%), Arkansas (74.2\%), Wyoming (73.2\%), South Dakota (72.5\%), Connecticut $(72.2 \%)$, and South Carolina (70.2\%). Only 1 state, Alaska (44.5\%), had a daily intake prevalence below 50.0\% (Table 2). Most states had a daily intake prevalence between $50.0 \%$ and $70.0 \%$ (Figure).

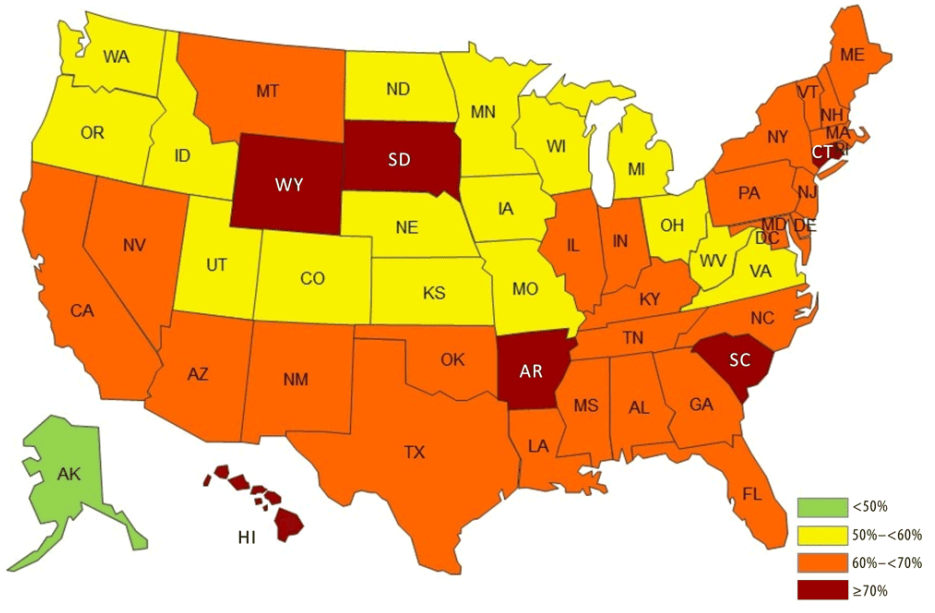

Figure. Prevalence of self-reported sugar-sweetened beverage (SSB) intake once daily or more among US adults by state, National Health Interview Survey Cancer Control Supplement (NHIS CCS), 2010 and 2015. SSBs include regular soda, sweetened fruit drinks, sports/energy drinks, and sweetened coffee/tea drinks. This map shows combined 2010 and 2015 data from the NHIS CCS $(9,10)$.

\section{Discussion}

Daily SSB intake is common among US adults and is particularly high in some states and among some populations. The prevalence in our study was higher than in the 2017 Behavioral Risk Factor Surveillance System (BRFSS) survey (8). This discrepancy may be explained by differences in the types of SSBs assessed, modes of survey administration, methods of collecting dietary intake data, and representativeness. Previous NHIS, NHANES (National Health and Nutrition Examination Survey), and BRFSS data also showed that SSB consumption is higher among young adults, men, adults in nonmetropolitan counties, and people with low levels of education $(6-8,11)$.

The prevalence of SSB consumption in previous studies was high in the Northeast (7) and in southern states (6), consistent with our study's findings. The high northeastern prevalence may be due to high consumption of sweetened coffee or tea drinks (7). Data from the 2017 BRFSS survey (8) for 12 states, and data from the 2013 BRFSS survey (6) for 23 states also revealed state-specific differences in SSB intake. Reasons for state differences may reflect demographic differences. States and communities may also differ in SSB marketing (12), pricing, and access to alternatives.

Our study has several limitations, including self-reported information, assessment of intake frequency without volume or amount of SSBs, age of the data, and combination of data. Declines in SSB intake have occurred over time (13). Combining data may mask changes in prevalence in the study period. Regardless, ours is the

\footnotetext{
The opinions expressed by authors contributing to this journal do not necessarily reflect the opinions of the U.S. Department of Health and Human Services, the Public Health Service, the Centers for Disease Control and Prevention, or the authors' affiliated institutions.
} 
first study to our knowledge to examine SSB intake frequency for all 50 states and the District of Columbia by using a nationally representative sample of US adults. Our findings highlight that prevalence of daily SSB intake remains high among US adults, with sociodemographic and geographic differences. Efforts to decrease SSB intake could consider the higher intake prevalence in sociodemographic and geographic subpopulations to aid design and targeting of equitable interventions.

\section{Acknowledgments}

We thank Wajun Cui from the National Center for Health Statistics, Division of Research and Methodology, and the staff of the NCHS Research Data Center. The findings and conclusions in this study are those of the authors and do not necessarily represent the official position of the Centers for Disease Control and Prevention. We received no funding for this study. No copyrighted materials were used in this article.

\section{Author Information}

Corresponding Author: Jennifer Chevinsky, MD, MPH, National Center for Chronic Disease Prevention and Health Promotion, Centers for Disease Control and Prevention, 4770 Buford Highway, NE, MS: S-107-5, Atlanta, GA 30341. Telephone: 404498-2890. Email: kst3@cdc.gov.

Author Affiliations: ${ }^{1}$ Division of Nutrition, Physical Activity, and Obesity, National Center for Chronic Disease Prevention and Health Promotion, Centers for Disease Control and Prevention, Atlanta, Georgia. ${ }^{2}$ Epidemic Intelligence Service, Centers for Disease Control and Prevention, Atlanta, Georgia.

\section{References}

1. Malik VS, Popkin BM, Bray GA, Després J-P, Hu FB. Sugarsweetened beverages, obesity, type 2 diabetes mellitus, and cardiovascular disease risk. Circulation 2010; 121(11):1356-64.

2. Malik VS, Hu FB. Fructose and cardiometabolic health: what the evidence from sugar-sweetened beverages tells us. J Am Coll Cardiol 2015;66(14):1615-24.

3. Bomback AS, Derebail VK, Shoham DA, Anderson CA, Steffen LM, Rosamond WD, et al. Sugar-sweetened soda consumption, hyperuricemia, and kidney disease. Kidney Int 2010;77(7):609-16.

4. Bernabé E, Vehkalahti MM, Sheiham A, Aromaa A, Suominen AL. Sugar-sweetened beverages and dental caries in adults: a 4-year prospective study. J Dent 2014;42(8):952-8.
5. Dietary Guidelines for Americans 2015-2020 8th Edition. 2015-2020 Dietary Guidelines. https://health.gov/our-work/ food-nutrition/previous-dietary-guidelines/2015. Accessed July 13, 2020.

6. Park S, Xu F, Town M, Blanck HM. Prevalence of sugarsweetened beverage intake among adults -23 states and the District of Columbia, 2013. MMWR Morb Mortal Wkly Rep 2016;65(7):169-74.

7. Park S, McGuire LC, Galuska DA. Regional differences in sugar-sweetened beverage intake among US adults. J Acad Nutr Diet 2015;115(12):1996-2002.

8. Imoisili O, Park S, Lundeen EA, Pan L, O'Toole T, Siegel KR, et al. Sugar-sweetened beverage intake among adults, by residence in metropolitan and nonmetropolitan counties in 12 states and the District of Columbia, 2017. Prev Chronic Dis 2020;17:E07.

9. National Cancer Institute. 2010 NHIS questionnaire - sample adult diet and nutrition. https://epi.grants.cancer.gov/diet/ shortreg/instruments/dsq-in-nhis-2010-english-version.pdf. Accessed March 16, 2021.

10. National Cancer Institute. 2015 NHIS questionnaire - sample adult diet and nutrition. https://epi.grants.cancer.gov/diet/ shortreg/instruments/dsq-in-nhis-ccs-2015-english-version.pdf. Accessed March 16, 2021.

11. Rosinger A, Herrick K, Gahche J, Park S. Sugar-sweetened beverage consumption among US adults, 2011-2014. NCHS Data Brief 2017;(270):1-8.

12. Martin-Biggers J, Yorkin M, Aljallad C, Ciecierski C, Akhabue I, McKinley J, et al. What foods are US supermarkets promoting? A content analysis of supermarket sales circulars. Appetite 2013;62:160-5.

13. Bleich SN, Vercammen KA, Koma JW, Li Z. Trends in beverage consumption among children and adults, 2003-2014. Obesity (Silver Spring) 2018;26(2):432-41.

The opinions expressed by authors contributing to this journal do not necessarily reflect the opinions of the U.S. Department of Health and Human Services, the Public Health Service, the Centers for Disease Control and Prevention, or the authors' affiliated institutions. 


\section{Tables}

Table 1. Prevalence of Sugar-Sweetened Beverage Intake Once Daily or More Among US Adults Aged 18 or Older ( $N=56,260)$, National Health Interview Survey Cancer Control Supplement, 2010 and $2015^{\mathrm{a}}$

\begin{tabular}{|c|c|c|}
\hline Characteristic & No. Respondents & $\geq 1$ Time/d, Weighted $\%(95 \% \mathrm{Cl})^{\mathrm{b}}$ \\
\hline Overall & 56,260 & $63.0(62.4-63.6)$ \\
\hline \multicolumn{3}{|l|}{ Age, $y^{b}$} \\
\hline $18-24$ & 5,358 & $65.0(63.3-66.7)$ \\
\hline $25-39$ & 15,027 & $65.4(64.4-66.3)$ \\
\hline $40-59$ & 19,143 & $62.8(61.8-63.7)$ \\
\hline$\geq 60$ & 16,732 & $59.7(58.6-60.8)$ \\
\hline \multicolumn{3}{|l|}{ Sex } \\
\hline Male & 25,148 & $66.1(65.3-67.0)$ \\
\hline Female & 31,112 & $60.0(59.3-60.8)$ \\
\hline \multicolumn{3}{|l|}{ Race/ethnicity ${ }^{b}$} \\
\hline White, non-Hispanic & 33,488 & $61.4(60.7-62.2)$ \\
\hline Black, non-Hispanic & 8,238 & $64.3(63.0-65.7)$ \\
\hline Hispanic & 9,984 & $70.1(68.7-71.4)$ \\
\hline Other, non-Hispanic & 4,550 & $60.5(58.5-62.5)$ \\
\hline \multicolumn{3}{|l|}{ Marital status } \\
\hline Married/domestic partnership & 28,079 & $62.7(61.9-63.4)$ \\
\hline Not married & 28,181 & $63.5(62.7-64.3)$ \\
\hline \multicolumn{3}{|l|}{ Education $^{b}$} \\
\hline$<$ High school & 8,712 & $69.8(68.5-71.0)$ \\
\hline High school/GED & 14,358 & $67.3(66.2-68.3)$ \\
\hline Some college & 17,200 & $62.8(61.8-63.8)$ \\
\hline College graduate & 15,990 & $56.4(55.4-57.4)$ \\
\hline \multicolumn{3}{|l|}{ Annual household income, $\$^{b}$} \\
\hline$<35,000$ & 23,665 & $66.0(65.2-66.9)$ \\
\hline $35,000-74,999$ & 17,061 & $64.3(63.3-65.3)$ \\
\hline
\end{tabular}

a Data are for 50 states and the District of Columbia. The type of SSBs consumed was based on survey respondents' answers to 4 questions: 1 ) "During the past month, how often did you drink regular soda or pop that contains sugar? Do not include diet soda."; 2) "During the past month, how often did you drink sweetened fruit drinks, such as Kool-Aid, cranberry, and lemonade? Include fruit drinks you made at home and added sugar to."; 3 ) "During the past month, how often did you drink sports and energy drinks such as Gatorade, Red Bull, and vitamin water?"; and 4) "During the past month, how often did you drink coffee or tea that had sugar or honey added to it? Include coffee and tea you sweetened yourself and presweetened tea and coffee drinks such as Arizona Iced Tea and Frappuccino. Do not include artificially sweetened coffee or diet tea."

${ }^{b}$ Significant difference in the prevalence of SSB intake once daily or more across levels of the characteristic at the $P<.05$ level based on $\chi^{2}$ test.

${ }^{c}$ Based on National Center for Health Statistics Urban-Rural Classification Scheme for Counties (https://www.cdc.gov/nchs/data_access/urban_rural.htm). Metropolitan includes large central metro, large fringe metro, medium metro, and small metro categories. Nonmetropolitan includes micropolitan and noncore categories.

d US Census Bureau-defined regions: Northeast (Connecticut, Maine, Massachusetts, New Hampshire, New Jersey, New York, Pennsylvania, Rhode Island, Vermont); Midwest (Illinois, Indiana, lowa, Kansas, Michigan, Minnesota, Missouri, Nebraska, North Dakota, Ohio, South Dakota, Wisconsin); Southern (Alabama, Arkansas; Delaware, District of Columbia, Florida, Georgia, Kentucky, Louisiana, Maryland, Mississippi, North Carolina, Oklahoma, South Carolina, Tennessee, Texas, Virginia, West Virginia); and Western (Alaska, Arizona, Colorado, Hawaii, Idaho, Montana, Nevada, New Mexico, Oregon, Utah, Washington, Wyoming). 
(continued)

Table 1. Prevalence of Sugar-Sweetened Beverage Intake Once Daily or More Among US Adults Aged 18 or Older ( $N=56,260)$, National Health Interview Survey Cancer Control Supplement, 2010 and $2015^{\mathrm{a}}$

\begin{tabular}{|c|c|c|}
\hline Characteristic & No. Respondents & $\geq 1$ Time/d, Weighted $\%(95 \% \mathrm{Cl})^{\mathrm{b}}$ \\
\hline $75,000-99,999$ & 5,744 & $61.8(60.1-63.4)$ \\
\hline$\geq 100,000$ & 9,790 & $57.7(56.4-59.0)$ \\
\hline \multicolumn{3}{|c|}{ Metropolitan/nonmetropolitan status ${ }^{\mathrm{b}, \mathrm{c}}$} \\
\hline Metropolitan & 46,623 & $62.7(62.0-63.3)$ \\
\hline Nonmetropolitan & 9,637 & $65.0(63.2-66.7)$ \\
\hline \multicolumn{3}{|l|}{ Census region $^{b, d}$} \\
\hline Northeast & 9,084 & $67.0(65.5-68.4)$ \\
\hline Midwest & 12,100 & $58.3(57.0-59.7)$ \\
\hline South & 20,072 & $65.2(64.2-66.1)$ \\
\hline West & 15,004 & $61.1(59.9-62.2)$ \\
\hline
\end{tabular}

a Data are for 50 states and the District of Columbia. The type of SSBs consumed was based on survey respondents' answers to 4 questions: 1 ) 'During the past month, how often did you drink regular soda or pop that contains sugar? Do not include diet soda."; 2) "During the past month, how often did you drink sweetened fruit drinks, such as Kool-Aid, cranberry, and lemonade? Include fruit drinks you made at home and added sugar to."; 3) "During the past month, how often did you drink sports and energy drinks such as Gatorade, Red Bull, and vitamin water?"; and 4) "During the past month, how often did you drink coffee or tea that had sugar or honey added to it? Include coffee and tea you sweetened yourself and presweetened tea and coffee drinks such as Arizona Iced Tea and Frappuccino. Do not include artificially sweetened coffee or diet tea."

${ }^{b}$ Significant difference in the prevalence of SSB intake once daily or more across levels of the characteristic at the $P<.05$ level based on $\chi^{2}$ test.

${ }^{c}$ Based on National Center for Health Statistics Urban-Rural Classification Scheme for Counties (https://www.cdc.gov/nchs/data_access/urban_rural.htm). Metropolitan includes large central metro, large fringe metro, medium metro, and small metro categories. Nonmetropolitan includes micropolitan and noncore categories.

'US Census Bureau-defined regions: Northeast (Connecticut, Maine, Massachusetts, New Hampshire, New Jersey, New York, Pennsylvania, Rhode Island, Vermont); Midwest (Illinois, Indiana, lowa, Kansas, Michigan, Minnesota, Missouri, Nebraska, North Dakota, Ohio, South Dakota, Wisconsin); Southern (Alabama, Arkansas; Delaware, District of Columbia, Florida, Georgia, Kentucky, Louisiana, Maryland, Mississippi, North Carolina, Oklahoma, South Carolina, Tennessee, Texas, Virginia, West Virginia); and Western (Alaska, Arizona, Colorado, Hawaii, Idaho, Montana, Nevada, New Mexico, Oregon, Utah, Washington, Wyoming).

The opinions expressed by authors contributing to this journal do not necessarily reflect the opinions of the U.S. Department of Health and Human Services, the Public Health Service, the Centers for Disease Control and Prevention, or the authors' affiliated institutions. 
Table 2. Prevalence by State of Sugar-Sweetened Beverage Intake Once Daily or More Among US Adults Aged 18 or Older, National Health Interview Survey Cancer Control Supplement, 2010 and 2015

\begin{tabular}{|c|c|c|}
\hline State & No. Respondents & Weighted $\%(95 \% \mathrm{Cl})^{a}$ \\
\hline Nation overall & 56,260 & $63.0(62.4-63.6)$ \\
\hline Alabama & 813 & $65.0(60.2-69.6)$ \\
\hline Alaska & 469 & $44.5(40.3-48.8)$ \\
\hline Arizona & 898 & $64.5(59.6-69.1)$ \\
\hline Arkansas & 602 & $74.2(70.2-77.8)$ \\
\hline California & 6,628 & $62.7(61.0-64.3)$ \\
\hline Colorado & 882 & $59.4(55.0-63.6)$ \\
\hline Connecticut & 652 & $72.2(67.8-76.3)$ \\
\hline Delaware & 463 & $68.0(60.5-74.6)$ \\
\hline District of Columbia & 563 & $64.8(57.5-71.4)$ \\
\hline Florida & 3,184 & $67.2(65.2-69.2)$ \\
\hline Georgia & 1,548 & $68.1(65.1-70.9)$ \\
\hline Hawaii & 516 & 76.4 (73.9-78.7) \\
\hline Idaho & 531 & $58.8(55.0-62.5)$ \\
\hline Illinois & 1,946 & $62.7(59.5-65.8)$ \\
\hline Indiana & 1,034 & $65.7(61.0-70.2)$ \\
\hline lowa & 752 & $50.5(44.3-56.7)$ \\
\hline Kansas & 815 & $54.9(51.5-58.3)$ \\
\hline Kentucky & 893 & $67.2(62.0-72.0)$ \\
\hline Louisiana & 787 & $68.7(65.2-71.9)$ \\
\hline Maine & 638 & $65.5(63.6-67.3)$ \\
\hline Maryland & 830 & $65.4(61.3-69.3)$ \\
\hline Massachusetts & 858 & $66.8(62.7-70.7)$ \\
\hline Michigan & 1,437 & $59.0(55.1-62.8)$ \\
\hline Minnesota & 985 & $50.4(46.2-54.7)$ \\
\hline Mississippi & 674 & $64.5(61.8-67.0)$ \\
\hline Missouri & 871 & $59.1(55.4-62.7)$ \\
\hline Montana & 467 & $64.9(63.4-66.3)$ \\
\hline Nebraska & 614 & $58.0(54.6-61.3)$ \\
\hline Nevada & 760 & $63.8(58.4-68.8)$ \\
\hline New Hampshire & 526 & $69.7(66.9-72.3)$ \\
\hline New Jersey & 1,220 & $69.5(65.6-73.2)$ \\
\hline New Mexico & 728 & $68.5(65.8-71.1)$ \\
\hline
\end{tabular}

a The type of SSBs consumed was based on survey respondents' answers to 4 questions: 1) "During the past month, how often did you drink regular soda or pop that contains sugar? Do not include diet soda."; 2) "During the past month, how often did you drink sweetened fruit drinks, such as Kool-Aid, cranberry, and lemonade? Include fruit drinks you made at home and added sugar to."; 3) "During the past month, how often did you drink sports and energy drinks such as Gatorade, Red Bull, and vitamin water?"; and 4) "During the past month, how often did you drink coffee or tea that had sugar or honey added to it? Include coffee and tea you sweetened yourself and presweetened tea and coffee drinks such as Arizona Iced Tea and Frappuccino. Do not include artificially sweetened coffee or diet tea."

The opinions expressed by authors contributing to this journal do not necessarily reflect the opinions of the U.S. Department of Health and Human Services, the Public Health Service, the Centers for Disease Control and Prevention, or the authors' affiliated institutions. 
(continued)

Table 2. Prevalence by State of Sugar-Sweetened Beverage Intake Once Daily or More Among US Adults Aged 18 or Older, National Health Interview Survey Cancer Control Supplement, 2010 and 2015

\begin{tabular}{|c|c|c|}
\hline State & No. Respondents & Weighted \% $(95 \% \mathrm{Cl})^{\mathrm{a}}$ \\
\hline New York & 2,701 & $65.6(63.1-68.1)$ \\
\hline North Carolina & 1,511 & $62.7(59.0-66.2)$ \\
\hline North Dakota & 506 & $59.2(53.8-64.5)$ \\
\hline Ohio & 1,716 & $57.2(54.1-60.3)$ \\
\hline Oklahoma & 669 & $66.0(59.1-72.3)$ \\
\hline Oregon & 708 & $51.5(48.6-54.4)$ \\
\hline Pennsylvania & 1,727 & $65.9(62.6-69.0)$ \\
\hline Rhode Island & 390 & $65.7(58.1-72.6)$ \\
\hline South Carolina & 739 & $70.2(64.6-75.4)$ \\
\hline South Dakota & 515 & $72.5(69.0-75.7)$ \\
\hline Tennessee & 909 & $66.4(61.2-71.2)$ \\
\hline Texas & 4,227 & $62.5(60.3-64.6)$ \\
\hline Utah & 734 & $53.6(49.1-58.1)$ \\
\hline Vermont & 372 & $67.3(64.6-69.8)$ \\
\hline Virginia & 1,097 & $59.6(56.1-63.0)$ \\
\hline Washington & 1,185 & $55.0(51.9-58.0)$ \\
\hline West Virginia & 563 & $59.4(55.8-62.9)$ \\
\hline Wisconsin & 909 & $50.4(46.6-54.2)$ \\
\hline Wyoming & 498 & $73.2(67.7-78.0)$ \\
\hline
\end{tabular}

a The type of SSBs consumed was based on survey respondents' answers to 4 questions: 1 ) "During the past month, how often did you drink regular soda or pop that contains sugar? Do not include diet soda."; 2) "During the past month, how often did you drink sweetened fruit drinks, such as Kool-Aid, cranberry, and lemonade? Include fruit drinks you made at home and added sugar to."; 3) "During the past month, how often did you drink sports and energy drinks such as Gatorade, Red Bull, and vitamin water?"; and 4) "During the past month, how often did you drink coffee or tea that had sugar or honey added to it? Include coffee and tea you sweetened yourself and presweetened tea and coffee drinks such as Arizona Iced Tea and Frappuccino. Do not include artificially sweetened coffee or diet tea."

The opinions expressed by authors contributing to this journal do not necessarily reflect the opinions of the U.S. Department of Health and Human Services, the Public Health Service, the Centers for Disease Control and Prevention, or the authors' affiliated institutions. 